\title{
Derivation and Application of Six-Point Linear Multistep Numerical Method for Solution of Second Order Initial Value Problems
}

\author{
Awari, Y. Sani \\ Department of Mathematics/Statistics Bingham University, Km 26 Keffi-Abuja expressway, P.M.B 005 Karu, \\ Nigeria
}

\begin{abstract}
A six-step Continuous Block method of order $(5,5,5,5,5,5)^{T}$ is proposed for direct solution of the second $\left(2^{\text {nd }}\right)$ order initial value problems. The main method and additional ones are obtained from the same continuous interpolant derived through interpolation and collocation procedures. The methods are derived by interpolating the continuous interpolant at $x=x_{n+j}, j=6$ and collocating the first and second derivative of the continuous interpolant at $x_{n+j}, j=0$ and $j=2,3, \ldots 5$ respectively. The stability properties of the methods are discussed and the stability region shown. The methods are then applied in block form as simultaneous numerical integrators. Two numerical experiments are given to illustrate the efficiency of the new methods.
\end{abstract}

Keywords: Collocation and Interpolation, Second Order Equations, Block Method, Initial Value Problem.

\section{Introduction}

In this paper, efforts are directed towards constructing a uniform order 5 block methods for solution of general second order ordinary differential equation of the form.

$y^{\prime \prime}=f\left(x, y, y^{\prime}\right), y(0)=\propto_{1} \quad y^{\prime}(0)=\beta$

In the past, efforts have been made by eminent scholars to solve higher order initial value problems especially the second order ordinary differential equation. In practice, this class of problem (1) is usually reduced to system of first order differential equation and numerical methods for first order Odes then employ to solve them, Fatunla (1988) and Lambert (1973). Awoyemi (1999) showed that reduction of higher order equations to its first order has a serious implication in the results; hence it is necessary to modify existing algorithms to handle directly this class of problem (1). Yahaya and Badmus (2009) demonstrated a successful application of LMM methods to solve directly a general second order odes of the form (1) though with non-uniform order member block method, this idea is used and now extended to our own uniform order block schemes to solve the type (1) directly.

We approximate the exact solution $\mathrm{y}(\mathrm{x})$ by seeking the continuous method $\bar{y}(x)$ of the form

$\bar{y}(x)=\sum_{j=o}^{s-1} \propto_{j}(x) y_{n+j}+h^{2} \sum_{j=o}^{r-1} \beta_{j}(x) f_{n+j}$

Where $x \in[a, b]$ and the following notations are introduced. The positive integer $k \geq 2$ denotes the step number of the method (2), which is applied directly to provide the solution to (1).

\section{Derivation Of The New Block Methods}

We propose an approximate solution to (1) in the form:

$y_{k}(x)=\sum_{j=0}^{s+r-1} V_{j} x^{j}$

$y_{k}^{\prime}(x)=\sum_{j=0}^{s+r-1} j V_{j} x^{(j-1)}$

$y_{k}^{\prime \prime}(x)=\sum_{j=0}^{s+r-1} j(j-1) V_{j} x^{(j-2)}=f\left(x, y, y^{\prime}\right)$

Now, interpolating (3) at $x_{n+j}, j=6$ and collocating (5) at $x_{n+j}, j=2,3, \ldots, 5$ leads to a system of equations written in the form.

$$
\begin{gathered}
V_{0}+V_{1} x_{n}+V_{2} x_{n}^{2}+V_{3} x_{n}^{3}+V_{4} x_{n}^{4}+V_{5} x_{n}^{5}+V_{6} x_{n}^{6}=y_{n} \\
V_{0}+V_{1} x_{n+1}+V_{2} x_{n+1}^{2}+V_{3} x_{n+1}^{3}+V_{4} x_{n+1}^{4}+V_{5} x_{n+1}^{5}+V_{6} x_{n+1}^{6}=y_{n+1} \\
V_{0}+V_{1} x_{n+2}+V_{2} x_{n+2}^{2}+V_{3} x_{n+2}^{3}+V_{4} x_{n+2}^{4}+V_{5} x_{n+2}^{5}+V_{6} x_{n+2}^{6}=y_{n+2} \\
V_{0}+V_{1} x_{n+3}+V_{2} x_{n+3}^{2}+V_{3} x_{n+3}^{3}+V_{4} x_{n+3}^{4}+V_{5} x_{n+3}^{5}+V_{6} x_{n+3}^{6}=y_{n+3} \\
V_{0}+V_{1} x_{n+4}+V_{2} x_{n+4}^{2}+V_{3} x_{n+4}^{3}+V_{4} x_{n+4}^{4}+V_{5} x_{n+4}^{5}+V_{6} x_{n+4}^{6}=y_{n+4} \\
V_{0}+V_{1} x_{n+5}+V_{2} x_{n+5}^{2}+V_{3} x_{n+5}^{3}+V_{4} x_{n+5}^{4}+V_{5} x_{n+5}^{5}+V_{6} x_{n+5}^{6}=y_{n+5} \\
2 V_{2}+6 V_{3} x_{n+6}+12 V_{4} x_{n+6}^{2}+20 V_{5} x_{n+6}^{3}+30 V_{6} x_{n+6}^{4}=f_{n+6}
\end{gathered}
$$

Where $V_{j}$ 's are the parameters to be determined.

When re-arranging (6) in a matrix form $A X=B$, we obtained 


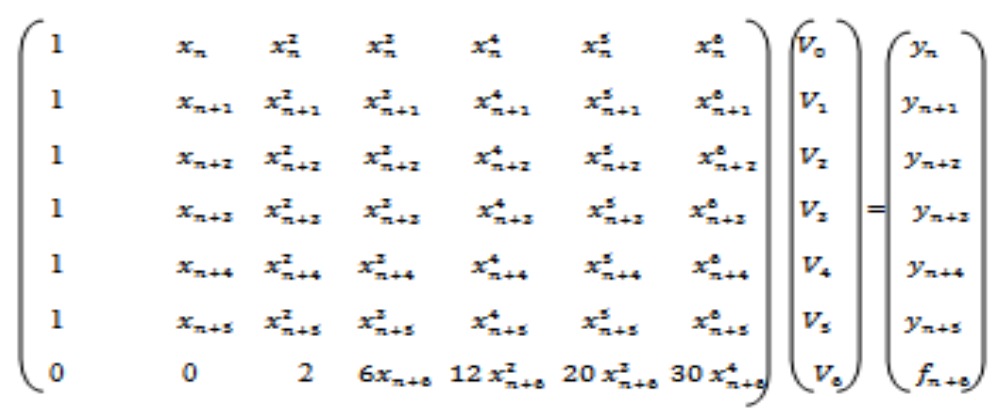

$y(x)=$

$$
\begin{aligned}
& q:=\left(\frac{-\frac{58997}{24360} x+\frac{58997}{24360} x_{n}}{h}+\frac{14235}{6496} \frac{\xi^{2}}{h^{2}}-\frac{37733}{38976} \frac{\xi^{3}}{h^{3}}\right. \\
& \left.+\frac{2899}{12992} \frac{\xi^{4}}{h^{4}}-\frac{4999}{194880} \frac{\xi^{5}}{h^{5}}+1+\frac{15}{12992} \frac{\xi^{6}}{h^{6}}\right) y_{n} \\
& +\left(\frac{\frac{2355}{406} x-\frac{2355}{406} x_{n}}{h}-\frac{11209}{9744} \frac{\xi^{4}}{h^{4}}+\frac{43451}{9744} \frac{\xi^{3}}{h^{3}}\right. \\
& \left.-\frac{13389}{1624} \frac{\xi^{2}}{h^{2}}-\frac{65}{9744} \frac{\xi^{6}}{h^{6}}+\frac{1381}{9744} \frac{\xi^{5}}{h^{5}}\right) y_{n+1} \\
& +\left(\frac{-\frac{5595}{812} x+\frac{5595}{812} x_{n}}{h}+\frac{307}{19488} \frac{\xi^{6}}{h^{6}}+\frac{42981}{3248} \frac{\xi^{2}}{h^{2}}\right. \\
& \left.-\frac{164891}{19488} \frac{\xi^{3}}{h^{3}}+\frac{47207}{19488} \frac{\xi^{4}}{h^{4}}-\frac{6229}{19488} \frac{\xi^{5}}{h^{5}}\right) y_{n+2}+( \\
& -\frac{9525}{812} \frac{\xi^{2}}{h^{2}}+\frac{\frac{3425}{609} x-\frac{3425}{609} x_{n}}{h}-\frac{4259}{1624} \frac{\xi^{4}}{h^{4}} \\
& \left.+\frac{40819}{4872} \frac{\xi^{3}}{h^{3}}-\frac{31}{1624} \frac{\xi^{6}}{h^{6}}+\frac{1801}{4872} \frac{\xi^{5}}{h^{5}}\right) y_{n+3} \\
& +\left(\frac{37563}{6496} \frac{\xi^{2}}{h^{2}}-\frac{170309}{38976} \frac{\xi^{3}}{h^{3}}+\frac{-\frac{4335}{1624} x+\frac{4335}{1624} x_{n}}{h}\right. \\
& \left.-\frac{8539}{38976} \frac{\xi^{5}}{h^{5}}+\frac{57049}{38976} \frac{\xi^{4}}{h^{4}}+\frac{461}{38976} \frac{\xi^{6}}{h^{6}}\right) y_{n+4}+( \\
& -\frac{69}{56} \frac{\xi^{2}}{h^{2}}+\frac{\frac{39}{70} x-\frac{39}{70} x_{n}}{h}+\frac{323}{336} \frac{\xi^{3}}{h^{3}}-\frac{113}{336} \frac{\xi^{4}}{h^{4}} \\
& \left.-\frac{1}{336} \frac{\xi^{6}}{h^{6}}+\frac{89}{1680} \frac{\xi^{5}}{h^{5}}\right) y_{n+5}+\left(\left(-\frac{15}{406} x+\frac{15}{406} x_{n}\right) h\right. \\
& +\frac{85}{3248} \frac{\xi^{4}}{h^{2}}-\frac{15}{3248} \frac{\xi^{5}}{h^{3}}+\frac{1}{3248} \frac{\xi^{6}}{h^{4}}-\frac{225}{3248} \frac{\xi^{3}}{h} \\
& \left.+\frac{137}{1624} \xi^{2}\right) f_{n+6}
\end{aligned}
$$

Where $V_{j}$ 's are obtained as continuous coefficients of $\propto_{j}(x)$ and $\beta_{j}(x)$

Specifically, from (2) the proposed solution takes the form 
$y(x)=\propto_{0}(x) y_{n}+\propto_{1}(x) y_{n+1}+\propto_{2}(x) y_{n+2}+\propto_{3}(x) y_{n+3}+\propto_{4}(x) y_{n+4}+\propto_{5}(x) y_{n+5}+$ $h^{2}\left[\beta_{6}(x) f_{n+6}\right]$

A mathematical software (maple) is used to obtained the inverse of the matrix in equation (7) were values for $V_{j}$ 's were established. After some manipulation to the inverse, we obtained the continuous interpolant of the form:

(9)

Evaluating (9) at $x_{n+j}, j=6$ and its second derivative evaluated at $x_{n+j}, j=2, \ldots 5$ while its $1^{\text {st }}$ derivative is evaluated at $x_{n+j}, j=0$ yields the following set of discrete equations.

$$
\begin{gathered}
\frac{29}{45} y_{n+6}-\frac{783}{315} y_{n+5}+\frac{1053}{252} y_{n+4}-\frac{254}{63} y_{n+3}+\frac{1485}{630} y_{n+2}-\frac{243}{315} y_{n+1}+\frac{137}{1260} y_{n}=\frac{h^{2}}{7}\left[f_{n+6}\right] \\
\frac{5162}{3} y_{n+5}-\frac{61891}{12} y_{n+4}+\frac{17960}{3} y_{n+3}-\frac{21277}{6} y_{n+2}+\frac{3478}{3} y_{n+1}-\frac{1955}{12} y_{n}=h^{2}\left[812 f_{n+5}-137 f_{n+6}\right] \\
2407 y_{n+5}-\frac{18173}{4} y_{n+4}+1606 y_{n+3}+\frac{1693}{2} y_{n+2}-373 y_{n+1}+\frac{227}{4} y_{n}=h^{2}\left[39 f_{n+6}+2436 f_{n+4}\right] \\
\frac{87}{2} y_{n+5}-\frac{2319}{3} y_{n+4}+1077 y_{n+3}-\frac{1185}{2} y_{n+2}+\frac{111}{2} y_{n+1}-\frac{15}{4} y_{n}=h^{2}\left[f_{n+6}-406 f_{n+3}\right] \\
\frac{29}{3} y_{n+5}-\frac{55}{12} y_{n+4}-\frac{1438}{3} y_{n+3}+\frac{5783}{6} y_{n+2}-\frac{1559}{3} y_{n+1}+\frac{361}{12} y_{n}=h^{2}\left[f_{n+6}-406 f_{n+2}\right] \\
\frac{377}{25} y_{n+5}-\frac{289}{4} y_{n+4}+\frac{5480}{36} y_{n+3}-\frac{373}{2} y_{n+2}+157 y_{n+1}-\frac{58997}{900} y_{n}-\frac{406}{15} h Z_{n}=h^{2} f_{n+6}
\end{gathered}
$$

Where $Z_{n}=y_{n}^{\prime}$

Equation (10) is the proposed six-step block method. The application of the block integrators (10) with $\mathrm{n}=0$, give the values of $\mathrm{y}_{1}, \mathrm{y}_{2}, \mathrm{y}_{3}, \mathrm{y}_{4}, \mathrm{y}_{5}$ and $\mathrm{y}_{6}$ directly without the use of starting values. I

\section{Order and error constant}

\section{Analysis of the Method}

Following Fatunla [5, 6 and 7] and Lambert [9 and 10] we define the local truncation error associated with the conventional form of (2) to be the linear difference operator.

$L[y(x) ; h]=\sum_{j=o}^{k} \propto_{j} y(x+j h)-h^{2} \beta_{j} y^{\prime \prime}(x+j h)$

Where the constant coefficients $C_{q}, q=0,1 \ldots$ are given as follows:

$C_{q}=\sum_{j=o}^{k} \propto_{j}$

$C_{1}=\sum_{j=o}^{k} j \propto_{j}$

$C_{q}=\frac{1}{q !} \sum_{j=o}^{k} j^{q} \propto_{j}-q(q-1) \sum_{j=o}^{k} j^{q-2} \beta_{j}$

According to Henrici [ 10 ], we say that the method (2) has order P if

$$
C_{0}=C_{1}=C_{2}=\cdots=C_{p}=C_{p+1}=0, \quad C_{p+2} \neq 0
$$

Our calculations reveal that the Block methods (10) have uniform order $\mathrm{P}=5$ and error constant given by the vector $C_{7}=\left(-\frac{1}{10}, \frac{13077}{90},-\frac{1631}{30},-\frac{7}{10},-\frac{469}{10},-\frac{137}{30}\right)^{T}$

\section{Convergence}

The block methods shown in (10) can be represented by a matrix finite difference equation in the form:

$I Y_{w+1}=A Y_{w-1}+h^{2}\left[\beta_{1} F_{w+1}+\beta_{0} F_{w-1}\right]$

Where

And $\mathrm{w}=0,1,2, \ldots$ and $\mathrm{n}$ is the grid index

$$
\begin{aligned}
& Y_{w+1}=\left(y_{n+1}, \ldots, y_{n+6}\right)^{T}, \quad Y_{w-1}=\left(y_{n-5}, \ldots, y_{n}\right)^{T}, \\
& F_{w+1}=\left(F_{n+1}, \ldots, F_{n+6}\right)^{T}, \quad F_{w-1}=\left(F_{n-4}, \ldots, F_{n}\right)^{T},
\end{aligned}
$$


and $\mathrm{B}_{\mathrm{o}}=0$

$$
\begin{aligned}
& I=\left(\begin{array}{lllllll}
1 & 0 & 0 & 0 & 0 & 0 & 0 \\
0 & 1 & 0 & 0 & 0 & 0 & 0 \\
0 & 0 & 1 & 0 & 0 & 0 & 0 \\
0 & 0 & 0 & 1 & 0 & 0 & 0 \\
0 & 0 & 0 & 0 & 1 & 0 & 0 \\
0 & 0 & 0 & 0 & 0 & 1 & 0 \\
0 & 0 & 0 & 0 & 0 & 0 & 1
\end{array}\right) \\
& A=\left(\begin{array}{lllllll}
0 & 0 & 0 & 0 & 0 & 0 & 1 \\
0 & 0 & 0 & 0 & 0 & 0 & 1 \\
0 & 0 & 0 & 0 & 0 & 0 & 1 \\
0 & 0 & 0 & 0 & 0 & 0 & 1 \\
0 & 0 & 0 & 0 & 0 & 0 & 1 \\
0 & 0 & 0 & 0 & 0 & 0 & 1 \\
0 & 0 & 0 & 0 & 0 & 0 & 1
\end{array}\right)
\end{aligned}
$$

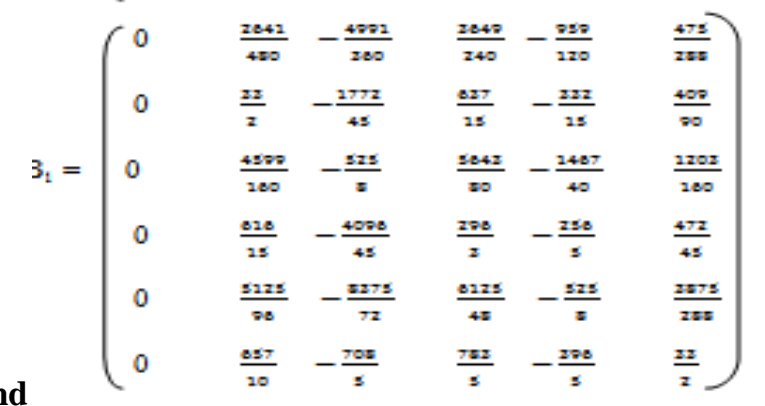

$$
\begin{aligned}
& -\frac{708}{5} \frac{783}{5} \quad \begin{array}{c}
\text { And } \\
-\frac{396}{5}
\end{array}
\end{aligned}
$$

tends to zero. Thus, as $h \rightarrow 0$, the method (13) tends to the difference system.

Whose first characteristic polynomial $\rho(Q)$ is given by

$$
I Y_{w+1}-A Y_{w-1}=0
$$

$=Q^{5}(Q-1)$

$$
\rho(Q)=\operatorname{det}(Q I-A)
$$

Following Fatunla [7], the block method (13) is zero-stable, since from (14), $\rho(Q)=0$ satisfy $\left|Q_{j}\right| \leq 1, j=$ $1, \ldots, k$ and for those roots with $\left|Q_{j}\right|=1$, the multiplicity does not exceed 2. The block method (13) is consistent as it has order $\mathrm{P}>1$. Accordingly following Henrici [8], we assert the convergence of the block method (13).

\section{Stability Region Of The Block Method}

To compute and plot absolute stability region of the block methods, the block method is reformulated as General Linear Methods expressed as:

$\left(\begin{array}{c}Y \\ y_{n+1}\end{array}\right)=\left(\begin{array}{ll}A & U \\ B & V\end{array}\right)\left(\begin{array}{c}h f(y) \\ y_{i-1}\end{array}\right)$

Where

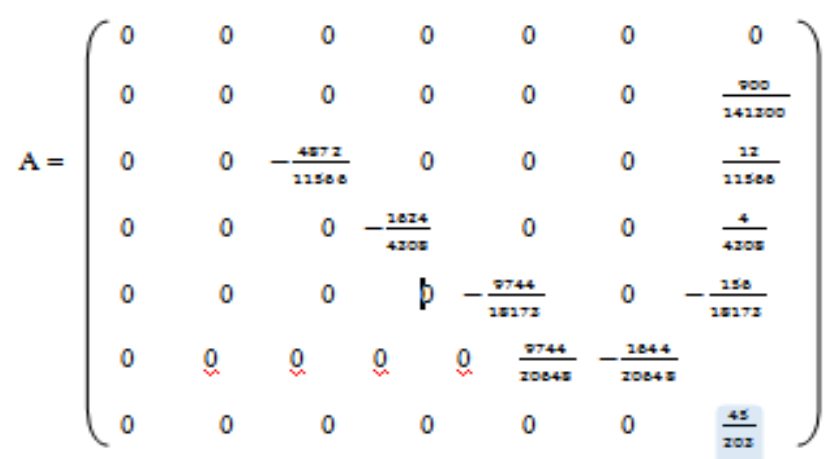




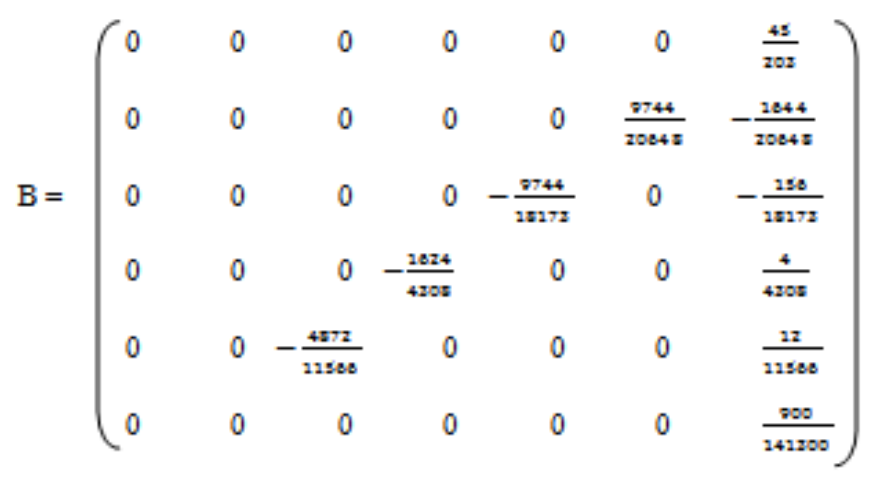

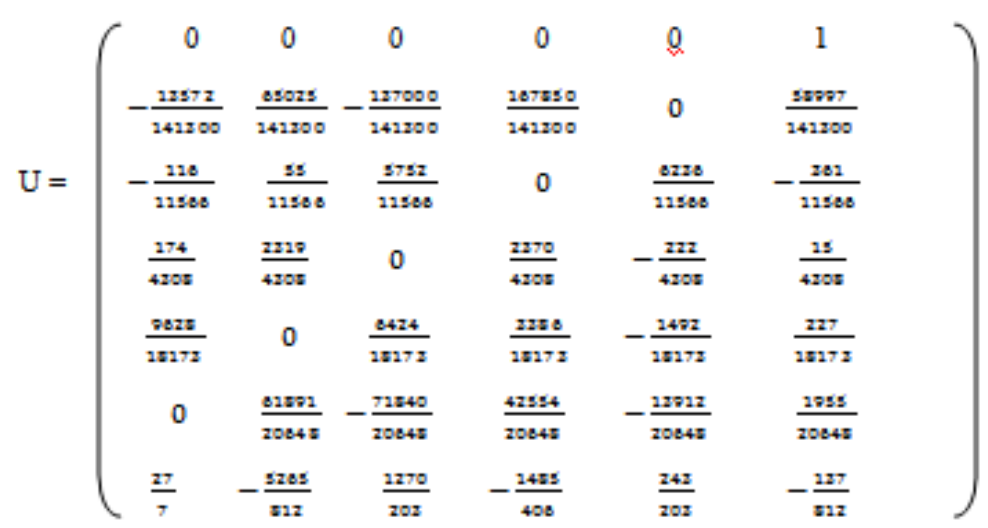



Substituting the values of $\mathrm{A}, \mathrm{B}, \mathrm{U}, \mathrm{V}$ into stability matrix and stability function,then using maple package yield the stability polynomial of the block method.Using a matlab program, we plot the absolute stability region of our proposed block method( see Fig. 1).

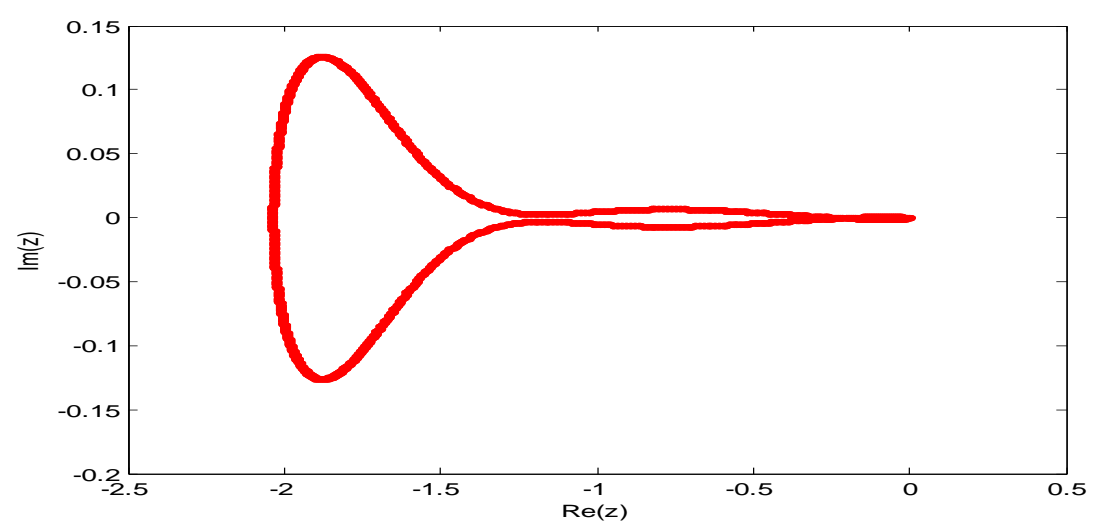

Fig. 1:Region of Absolute Stability 


\section{IMPLEMENTATION STRATEGIES}

In this section, we have tested the performance of our six-step block method on two (2) numerical problems by considering two IVPs (Initial Value Problems).For each example; we find the absolute errors of the approximate solution. Example 1.1: We consider the IVP for the step-size $h=0.01$

$$
y^{\prime \prime}-100 y=0, y(0)=1, y^{\prime}(0)=-10
$$

Table of results and absolute errors for problem 1.1

\begin{tabular}{|l|l|l|l|}
\hline \multicolumn{1}{|c|}{$x$} & \multicolumn{1}{|c|}{$y(x)$} & \multicolumn{1}{c|}{$y$} & \multicolumn{1}{c|}{ Errors } \\
\hline 0 & 1.0000000000 & 1.0000000000 & $0.00 \mathrm{e}+0$ \\
\hline 0.01 & 0.9048374180 & 0.9048372827 & $1.353 \mathrm{e}-7$ \\
\hline 0.02 & 0.8187307531 & 0.8187303873 & $3.658 \mathrm{e}-7$ \\
\hline 0.03 & 0.7408182207 & 0.7408176156 & $6.051 \mathrm{e}-7$ \\
\hline 0.04 & 0.6703200460 & 0.6703191958 & $8.502 \mathrm{e}-7$ \\
\hline 0.05 & 0.6065306597 & 0.6065295559 & $1.104 \mathrm{e}-6$ \\
\hline 0.06 & 0.5488116364 & 0.5488102673 & $1.369 \mathrm{e}-6$ \\
\hline 0.07 & 0.4965853038 & 0.4965838539 & $1.450 \mathrm{e}-6$ \\
\hline 0.08 & 0.4493289641 & 0.4493273672 & $1.597 \mathrm{e}-6$ \\
\hline 0.09 & 0.4065696597 & 0.4065678968 & $1.763 \mathrm{e}-6$ \\
\hline 0.10 & 0.3678794412 & 0.3678774948 & $1.946 \mathrm{e}-6$ \\
\hline 0.11 & 0.3328710837 & 0.3328689844 & $2.099 \mathrm{e}-6$ \\
\hline 0.12 & 0.3011942119 & 0.3011918381 & $2.374 \mathrm{e}-6$ \\
\hline
\end{tabular}

Absolute Errors $|y(x)-y|$, for example 1.1, where $y(x)=e^{-10 x}$

Example 1.2: We consider the IVP for the step-size $h=0.1$

$$
y^{\prime \prime}+y=0, y(0)=1, y^{\prime}(0)=1
$$

Table of results and absolute errors for problem 1.2

\begin{tabular}{|l|l|l|l|}
\hline $\mathrm{X}$ & \multicolumn{1}{|c|}{$y(x)$} & \multicolumn{1}{|c|}{$y$} & \multicolumn{1}{c|}{ Errors } \\
\hline 0 & 1.0000000000 & 1.0000000000 & $0.00 \mathrm{e}-0$ \\
\hline 0.1 & 1.0948375819 & 1.0948374662 & $1.157 \mathrm{e}-7$ \\
\hline 0.2 & 1.1787359086 & 1.1787355987 & $3.099 \mathrm{e}-7$ \\
\hline 0.3 & 1.2508566958 & 1.2508561903 & $5.055 \mathrm{e}-7$ \\
\hline 0.4 & 1.3104793363 & 1.3104786406 & $6.957 \mathrm{e}-7$ \\
\hline 0.5 & 1.3570081005 & 1.3570072216 & $8.789 \mathrm{e}-7$ \\
\hline 0.6 & 1.3899780883 & 1.3899770347 & $1.054 \mathrm{e}-6$ \\
\hline 0.7 & 1.4090598745 & 1.4090588666 & $1.008 \mathrm{e}-6$ \\
\hline 0.8 & 1.4140628003 & 1.4140618777 & $9.226 \mathrm{e}-7$ \\
\hline 0.9 & 1.4049368779 & 1.4049360518 & $8.261 \mathrm{e}-7$ \\
\hline 1.0 & 1.3417732907 & 1.3417725691 & $7.216 \mathrm{e}-7$ \\
\hline 1.1 & 1.3448034815 & 1.3448028716 & $6.099 \mathrm{e}-7$ \\
\hline 1.2 & 1.2943968404 & 1.2943963485 & $4.919 \mathrm{e}-7$ \\
\hline
\end{tabular}

Absolute Errors $|y(x)-y|$, for example 1.2, where $y(x)=\operatorname{Cos} x+\operatorname{Sin} x$

\section{Conclusions}

We have proposed a six-step block LMM with continuous coefficients from which multiple finite difference methods were obtained and applied as simultaneous numerical integrators, without first adapting the ODE to an equivalent first order system. The method is derived through interpolation and collocation procedures by the matrix inverse approach. We conclude that our new six-step block method of uniform order 5 is suitable for direct solution of general second order differential equations. The new block methods are self- starting and all the discrete schemes used were obtained from the single continuous Formulation and its derivative which are of uniform order of accuracy. The results were obtained in block form which speeds up the computational process and the result obtained from the two numerical examples converges with the theoretical solutions.

\section{Reference}

[1] Aiken,R.C, Stiff Computation.New York, (Oxford:Oxford University Press ,1985).

[2] Atkinson,K.E, An introduction to Numerical Analysis,2nd Edition, (John Wiley and sons,New York ,1987).

[3] Awoyemi,D.O, On some continuous Linear Multistep Methods for Initial Value Problems,PhD.Thesis (Unpublished), University of Ilorin,Nigeria, (1992).

[4] Butcher,J.C , Numerical Methods for Ordinary differential systems, (John Wiley \& sons, west Sussex,England, 2003).

[5] Fatunla,S.O, Block methods for second order IVP's. Inter.J.Comp.Maths.72,No.1,(1991).

[6] Fatunla,S.O, Parallel methods for second order ODE's Computational ordinary differential equations, (1992).

[7] Fatunla,S.O, Higher order parallel methods for second order ODE's.Scientific Computing.Proceeding of fifth International Conference on Scientific Computing, (1994).

[8] Henrici,P., Discrete variable methods for ODE's.John Wiley, New York,(1962). 
[9] Lambert,J.D, Computational methods for ordinary differential equations.(John Wiley,New York, 1973).

[10] Lambert,J.D , Numerical methods for ordinary differential systems.John Wiley,New York ,(1991).

[11] Lie I and Norset,S.P, Super Convergence for Multistep Collocation.Math. Comp 52, (1989).

[12] Onimanyi,P.,Awoyemi,D.O.,Jator,S.N and Sirisena,U.W., New Linear Multistep Methods with continuous coefficients for first order initial value problems.J.Nig.Math.Soc.(1994).

[13] Onumanyi,P.,Sirisena,U.W and Jator,S.N., Continuous finite difference approximations for solving differential equations. Inter.J.Comp.Maths, .(1999). 